\section{Two-way ANOVA with and without repeated measurements, tests of simple main effects, and multiple comparisons for microcomputers}

\section{DANIEL COULOMBE \\ University of Ottawa, Ottawa, Ontario}

Many excellent programs to perform factorial analyses of variance (ANOVAs) are available to microcomputer users (Collani \& Waloszek, 1983; Corrigan, Bonelli, \& Borys, 1980a, 1980b; Coulombe, 1983; Galla, 1981; Hacker \& Angiolillo-Bent, 1981; Lane, 1981). Most of these can be used for the analysis of a variety of designs with equal or unequal numbers of subjects per cell. Only one of the above-mentioned packages allows for multiple comparisons with user-defined contrasts (Hacker \& Angiolillo-Bent, 1981). None can perform tests of simple main effects when there are significant interactions in factorial designs. The purpose of the programs described here is to fully analyze experimental data derived from two-factor designs with or without repeated measures. A full analysis should include not only the ANOVA table and a listing of the marginal and cell means, but also tests of simple main effects when the interaction is significant, multiple comparisons on marginal means for both factors, and multiple comparisons on cell means.

The present package includes three separate programs to perform two-way analysis of variance with no repeated measurements, or repeated measurements over one or two factors. Each program can be run on a microcomputer with a minimum of $16 \mathrm{~K}$ memory (RAM). The computational procedures used are described in Kirk (1968) and Winer (1971).

Input. Each program starts by requesting the number of levels for factor A and factor B. If independent samples are analyzed, the program (AOV2W) asks for the number of subjects in each cell (Ns can be unequal) and then accepts sequential entry of each score. If the analysis involves repeated measurements over one factor, the program (AOV2WRM1) requests the number of subjects in each level of factor A (the between factor). If the $\mathrm{Ns}$ are unequal, then the user is provided with the choice of using either an unweighted-means or a least-squares solution. Then each score is entered sequentially. Finally, if the design is completely crossed (repeated measurements over two factors), the program (AOV2WRM2) asks for the number of replications and proceeds with sequential entry of each score.

Output. A sample output of the AOV2WRM1 program is presented in Table 1. The outputs of the other programs follow a similar pattern. Each program output

The author is affiliated with the School of Psychology, University of Ottawa, Ottawa, Ontario, Canada K1N 6N5.
Table 1

Sample Output for AOV2WRM1

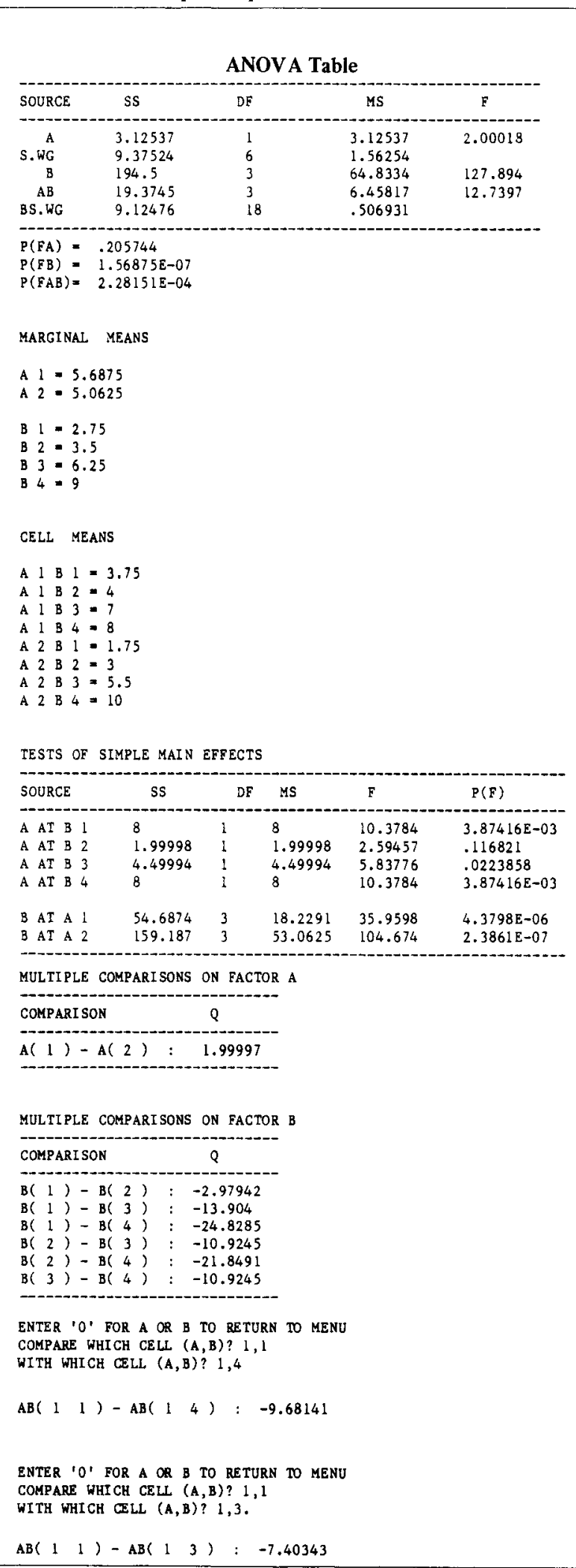


contains a complete ANOVA table, including the computed $\mathrm{F}$ values and their associated probabilities (Poole $\&$ Borchers, 1979). In each case, both the marginal and the interaction means are tabulated. Then the analysis of simple main effects is performed for both factors ( $A$ at $B_{j}$ and $B$ at $A_{i}$ ). Finally, the user can select a series of multiple comparisons on factor $\mathrm{A}$, factor $\mathrm{B}$, or their interaction. In this case, the program performs the computation of pairwise Tukey or Newman-Keuls tests and prints the resulting $Q$ values. The only task left to the user is to refer to the Studentized range statistics, found in most textbooks on experimental design, and determine which comparisons are statistically significant.

All outputs are directed to the line printer. To obtain screen output, the LPRINT statements should be changed to PRINT statements or to any routing instructions available on the system in use.

Limitations. The number of levels for each factor is dependent on the amount of RAM memory available in the system. Each program contains a DIM statement that can be changed to fit the user's needs. As distributed, AOV $2 W$ can analyze a $20 \times 20$ design with unlimited number of subjects per cell; AOV2WRM1 can be used to analyze a $15 \times 15$ design with 30 subjects per level of $A$; AOV2WRM2 can analyze a $15 \times 15$ design with 50 replications. These limits are for a $16 \mathrm{~K}$ microcomputer. If more memory is available, they can be raised accordingly.

Language and Computer. The programs were developed on a TRS-80 Model I, using Microsoft BASIC. They also have been tested with success on a TRS- 80 Model III and adapted easily for use on an IBM PC and Tandy 2000. The programs can be adapted for use on almost any computer equipped with a standard version of BASIC and at least $16 \mathrm{~K}$.
Availability. Program listings and documentation are available at no cost from Daniel Coulombe, School of Psychology, University of Ottawa, 275 Nicholas, Ottawa, Ontario, Canada K1N 6N5.

\section{REFERENCES}

Collani, G., von, \& Waloszek, G. (1983). UNIVARAN: A universal analysis of variance program. Behavior Research Methods \& Instrumentation, 15, 607.

Corrigan, J. C., Bonelli, P. J., \& Borys, S. V. (1980a). BASIC programs for one-way through four-way between-subjects and within-subjects ANOVAs. Behavior Research Methods \& Instrumentation, 12, 468.

Corrigan, J. C., Bonelli, P. J., \& Borys, S. V. (1980b). BASIC programs for two-way through four-way mixed-design ANOVAs. Behavior Research Methods \& Instrumentation, 12, 546.

Coulombe, D. (1983). BASIC programs for advanced statistical analysis. Ottawa: University of Ottawa Press.

GALLA, J. P. (1981). Factorial analysis of variance with unequal observations: A BASIC program for microcomputers. Behavior Research Methods \& Instrumentation, 13, 699-700.

Hacker, M. J., \& Angiolillo-Bent, J. S. (1981). A BASIC package for $\mathbf{N}$-way ANOVA with repeated measures, trend analysis, and user-defined contrasts. Behavior Research Methods \& Instrumentation, 13, 688.

KırK, R. E. (1968). Experimental design: Procedures for the behavioral sciences. Belmont, CA: Brooks/Cole.

LANE, D. M. (1981). A general analysis of variance program for microcomputers. Behavior Research Methods \& Instrumentation, 13, 694.

Poole, L., \& Borchers, M. (1979). Some common BASIC programs (3rd ed.) Berkeley, CA: Osborne/McGraw-Hill.

Wine R, B. J. (1971). Statistical principles in experimental designs. New York: McGraw-Hill.

(Manuscript accepted for publication June 26, 1984.) 